\title{
Encoding effects in one priming paradigm
}

\author{
WILMA A. WINNICK and RAYMOND PENKO \\ Queens College of the City University of New York, Flushing, New York
}

Four variations of a priming stimulus were introduced in three experiments, with the aim of examining the effects of encoding the prime on one target task (tachistoscopic recognition thresholds). The primes studied were words, color photographs, and outline drawings, followed by word and outline-drawing threshold measurement (Experiment 1); increased size and brightness reversal of words (Experiment 2); and mirror reversals of the prime (Experiment 3). Memory facilitation was weak or lacking for only the format changes in the first experiment. Facilitation was equal to that obtained with identical primes for the size and brightness alterations, and for the mirror reversals in Experiments 2 and 3. With weak or no facilitation in only one experiment, and strong facilitation in the others, we discuss the implications for the influence of encoding on priming effects.

Priming experiments use a second-stage task to uncover memory for materials previously presented to a subject, with no instructions to learn. These operations make the priming paradigm an ideal vehicle for the study of implicit memory. Within this two-stage framework, implicit memory is indicated if responses to materials experienced before (primed) are facilitated, compared with responses to new (control) items.

The two-stage format allows for the manipulation of potentially relevant variables at one or both stages. Encoding occurs at the input (priming) stage, and incoming material is put into a form compatible with storage. For implicit memory that occurs in the absence of instructions to learn and with automatic processing, encoding appears to play a minor role, involving merely the pronouncing or silent reading of the words presented.

In an early study not directly concerned with priming, Winnick and Nachbar (1967) alluded to the potential significance of encoding for a priming experiment. They found differences in second-stage tachistoscopic thresholds resulting from differences in the encoding of the stimulus $(S)$ and response $(R)$ items in a prior paired-associate learning task. In that study, the $S$ items were viewed and studied passively without verbalization, whereas the $\mathbf{R}$ items, which were seen for a somewhat shorter period of time (at least in the anticipation method), participated in anticipation and response production and effected reinforcement in the form of knowledge of results. Winnick and Nachbar attributed the lower second-stage thresholds for the $\mathrm{R}$ items, as compared with the $\mathrm{S}$ words, to differences in first-stage encoding. That first-stage encoding

This study was partially supported by a General Research Support Grant (NIH RR07064) to Queens College of the City University of New York. We are indebted to Leslie Glick and Stephanie Sandler for their assistance as experimenters. Requests for reprints should be sent to Wilma A. Winnick, Department of Psychology, Queens College of the City University of New York, Flushing, NY 11367. may influence implicit memory has been suggested in only a few studies of priming (e.g., Jacoby \& Hayman, 1987; Roediger \& Blaxton, 1987).

In the present experiments, some of the structural features of the prime were varied, with the aim of invoking different encoding activities predicted to affect subsequent threshold values.

\section{GENERAL METHOD}

The three experiments reported here used parallel materials, apparatus, procedures, and experimental designs. These features will be outlined here, with significant variations to be included in the description of each experiment.

\section{Materials and Apparatus}

The materials used for priming and threshold measurement in each experiment will be separately described. A Kodak projector, fitted with a timer and a voice key, was used in Experiment 3 to present the word primes and to time the latency of the identifying response. A two-channel Scientific Prototype tachistoscope measured tachistoscopic recognition thresholds in the second stage of each of the three experiments.

\section{Procedure}

Each experiment began with four familiarization trials with the tachistoscope, using materials of the kind to be used in the second stage (either words or outline drawings). The experimental materials were presented in the priming stage. The subjects were instructed to pronounce the words or to name the objects pictured (Experiment 1) as they were shown for $2 \mathrm{sec}$ each, by hand (Experiments 1 and 2), and by the projection tachistoscope (Experiment 3 ). In the test stage, the concepts previously seen, as well as new concepts, were presented in random order in altered form and in a form identical to the target, as typed words on cards, for threshold measurement. An ascending method of limits was used to determine these thresholds, which were the key measurements from each experiment.

\section{Experimental Design}

All three experiments employed a mixed design, with the major independent variable-the priming materials (identical, altered, and control)-represented within subjects. Counterbalanced replication groups were one independent groups variable in all three experiments. In addition, in Experiment 1 two groups differing in target materials were used, and in Experiment 2 two groups using different primes were studied. 


\section{EXPERIMENT 1}

Winnick and Daniel (1970) have suggested that subjects who have seen pictures of objects gain no advantage over controls when the objects' names follow and must be identified (an implicit memory task). In the present experiment, we investigated whether this finding holds true when word or picture primes are followed by threshold measurement of outline drawings. In other words, do all format changes operate similarly on all targets?

\section{Method}

Subjects. The subjects were 32 undergraduate volunteers, randomly assigned to groups, whose thresholds for either words or outline drawings were measured.

Materials and Apparatus. The selection of specific concepts to be used was based on line drawings of intermediate complexity from Snodgrass and Vanderwart (1980) which could be matched to magazine pictures. Corresponding names were either seven or eight letters in length.

For the measurement of outline-drawing thresholds, the outlines of Snodgrass and Vanderwart (1980) were doubled in size; preliminary experimentation found that this size produced thresholds close to those for words.

\section{Results}

Thresholds for the two groups (tested on either words or outline drawings) are shown in Figure 1. Thresholds were lowest for concepts primed in the same format in which they were tested, but some facilitation is also evident when outline drawings preceded word thresholds.

A 4 (priming materials) $\times 2$ (threshold groups) $\times 4$ (replications) mixed ANOVA of threshold scores revealed a significant effect of priming materials $[F(3,72)=9.61$, $p<.01$ ], which indicates facilitation by materials seen before (in any format) compared with control materials not seen previously. Of greater relevance is the significant groups $\times$ primes interaction $[F(3,72)=17.10$, $p<.01$, which suggests differential effects of the three kinds of primes on word and outline thresholds. The Scheffé significant differences (SSD) test revealed significantly lower thresholds when words were primed by words, than when words were primed by outlines or photographs $(p<.05)$. At the same time, words primed by outlines had significantly lower thresholds than controls $(p<.05)$, whereas thresholds of photographprimed words did not differ from the controls.

For outline-drawing thresholds, the SSD test indicated that priming by outline drawings produced significantly lower thresholds than those produced by the other primes $(p<.05)$. Photograph primes produced lower thresholds than did controls, whereas word primes produced higher thresholds than did controls; neither comparison was significant, however.

\section{Discussion}

One of the findings of this experiment confirms results that have been reported a number of times; that is, that prime-to-target format changes deter memory facilitation. The finding that photograph primes were not strong candidates for facilitation of word targets (and that words and photographs were not facilitative of outlines) joins a host of other findings that format shifts fail to produce memory facilitation in studies that used a variety of target tasks (e.g., Morton, 1979; Scarborough, Gerard, \& Cortese, 1979; Weldon \& Roediger, 1987). Such findings have been almost unanimous (an exception is the study by Sperber, McCauley, Ragain, \& Weil, 1979), which leads us to conclude that this failure of facilitation is a highly robust effect.

A somewhat novel finding is the facilitation of word thresholds by outline drawings (but not by photographs), without a parallel effect of

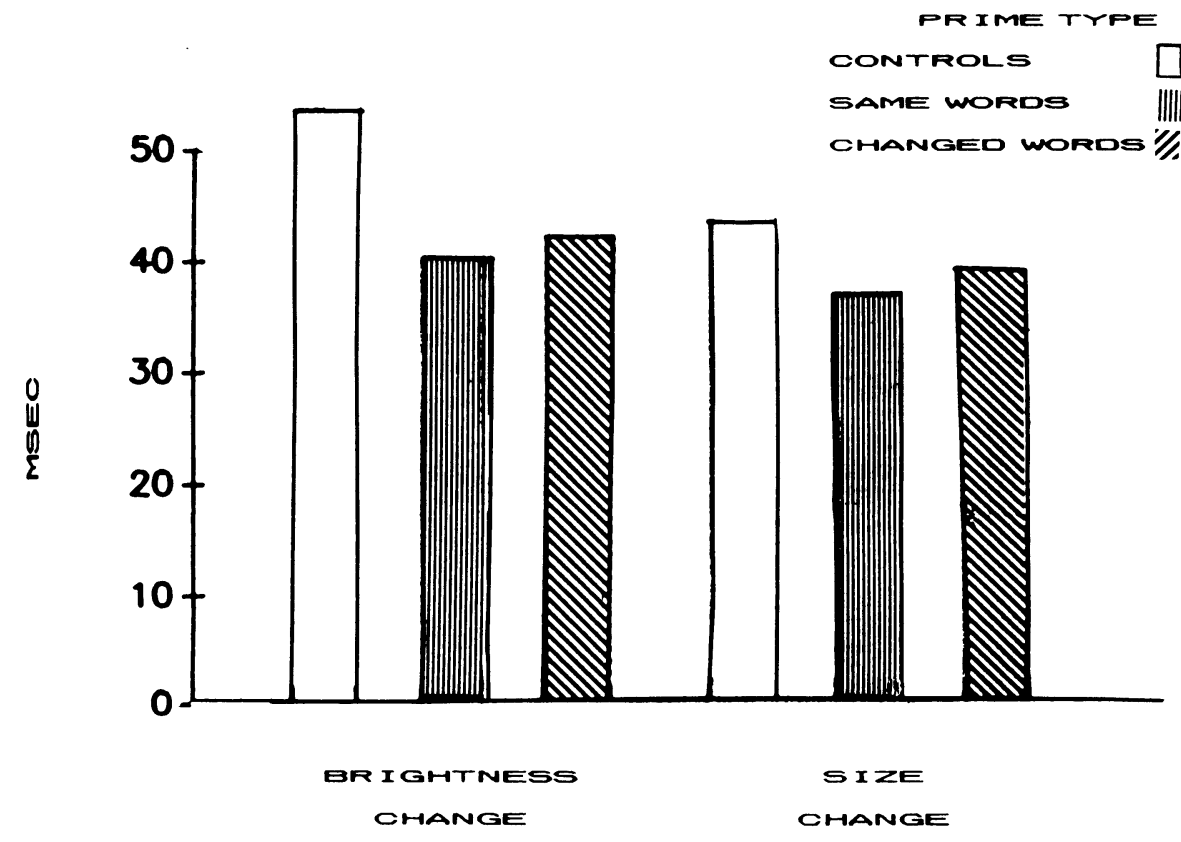

Figure 1. Mean recognition thresholds, from Experiment 1, for words (on the left) and for outline drawings (on the right) after priming with words, outline drawings, and colored photographs, compared to thresholds for controls. 
word primes on outline thresholds. In fact, word-primed outline drawings yielded thresholds even higher than did controls. Encoding tendencies at the first stage seem to be at work here; word primes that precede outline-drawing thresholds evoke images of the object that are unlikely to coincide with the features of the outline-drawing targets. When outline drawings prime words, on the other hand, the verbal identifying response to the depiction of a simple object transfers more readily.

Photograph priming of words seems to be hindered by responses to the myriad details present in a photograph. The possibility that simpler, less detailed pictorial materials (such as outlines) can serve as more transferable primes than can highly detailed ones has not been experimentally examined. Although Ritchey (1982) reported that outline drawings fared better in free recall than did photographs, his explanation for the difference is not applicable to priming.

The finding that color photographs used as primes produced little facilitation for outline-drawing thresholds is also of some relevance to an encoding analysis. It seems that the complexity of photographs evokes encoding responses that can make transfer to different second-stage representations difficult. The abundance of detail and the presence of color must produce encoding responses to these particular stimuli that are no more helpful for identification of outlines than for words. In a similar vein, Jacoby and Brooks (1984) found better identification of pictures that followed identical pictures than of those that followed samename pictures.

\section{EXPERIMENT 2}

In Experiment 2, two kinds of surface contrasts between primes and targets were examined. The primes used in two experimental groups were words that differed from targets either in letter size or in black-white contrast.

\section{Method}

Subjects. The subjects were $\mathbf{4 2}$ undergraduate volunteers, randomly assigned to two groups, and given either size- or brightness-altered primes.

Materials. The words presented as primes and for threshold measurement were 18 seven- and eight-letter words selected from Tulving, Schacter, and Stark (1982). These words were prepared on $3 \times 5$ in. cards in three ways: (1) the 18 words were typed in the same typescript as that used for threshold measurement, to serve as identical primes for both groups, and as controls; (2) for the size-altered group, the words were also prepared using Letraset with letters $7 \mathrm{~mm}$ high and $5 \mathrm{~mm}$ wide; (3) for the brightness-altered group, photographic negatives of the words provided the brightness reversal.

For first-stage priming, 12 of the words were seen by each group, half altered and half unchanged. For threshold measurement, all the words seen before the test and six control words were typed on $4 \times 5$ in. cards for presentation in the Scientific Prototype tachistoscope. Three replication groups were used to counterbalance the words across conditions.

\section{Results}

Both the size- and brightness-altered primes yielded second-stage thresholds as low as did the unchanged words: 38.9 and $39.7 \mathrm{msec}$ for the altered primes, and 36.8 and $40.3 \mathrm{msec}$ for the identical words in the two groups. Control thresholds for the two groups were 47.6 and $52.3 \mathrm{msec}$, respectively. A mixed ANOVA revealed a significant effect of prime type $[F(2,72)=39.9, p<$ $.01]$. The SSD test indicated that control thresholds were significantly higher than all others $(p<.05)$.

\section{Discussion}

In this experiment, we found two physical changes in the priming stimulus that failed to exert a damping effect on prime- target memory facilitation. The changes studied-an increase in letter size and brightness reversal-apparently left the word's overall visual impact intact; despite the changes, the prime and target words were seen as having the same letter shapes and overall configuration, encoding of the materials was unaffected, and identifying responses could be given readily and with a low threshold in the test stage.

\section{EXPERIMENT 3}

In this experiment, mirror-reversed words served as primes; this alteration reversed the spatial position of each letter and of the entire word.

\section{Method}

Subjects. The subjects were 42 undergraduate volunteers.

Materials. For priming, words were presented on slides, half in normal orientation and half as mirror reversals (accomplished by the orientation of the slides in the tray). The words used, 16 in number, were sixand seven-letter concrete nouns, all of high frequency. The word primes were presented by the Kodak projector described above, which also permitted measurement of response latencies.

\section{Results}

Response latencies at the priming stage. Mean response latencies for words seen in normal orientation were $0.86,0.77$, and $0.80 \mathrm{sec}$ in the three replications, respectively; for the mirror-reversed words, the means were $1.20,1.16$, and $1.39 \mathrm{sec}$, respectively. A mixed ANOVA revealed significantly longer latencies for the mirror-reversed primes than for words in normal orientation $[F(1,18)=42.00, p<.01]$.

Recognition thresholds. The mean tachistoscopic thresholds for words primed by normal, mirror-reversed, and control words were $34.6,36.1$, and $53.3 \mathrm{msec}$, respectively. A 2 (alteration groups) $\times 3$ (word types) $\times$ 3 (replications) mixed ANOVA revealed a significant effect only for the materials $[F(2,36)=5.94, p<.01]$, which clearly indicates that both the mirror-reversed and the normal primes produced significantly lower secondstage thresholds than did the control words.

\section{Discussion}

Two related findings from this experiment-the increased latency of mirror-reversed primes and the facilitation of target words primed by mirror reversals - suggest possible contributions from deep levels of processing and/or word generation combined with the imagery involved in problem solving.

It is unlikely that longer latencies during priming signal a deep level of processing for mirror-reversed primes. The operations required by reversal are not in accordance with Craik and Lockhart's (1972) analysis of processing levels. Craik and Lockhart described the deepest level of processing as impinging on the semantic features of the word; in the present experiment, meanings, referents, and associations to the words presented were clearly irrelevant to the subject's task. Rather, encoding mirror-reversed words entailed generation of the word by means of the imagery required to reverse the individual letters, as well as a degree of effort, as problem solving was necessary to produce the word. This suggests a strength-of-processing effect but not a levels effect (e.g., Tyler, Hertel, McCallum, \& Ellis, 1979).

\section{GENERAL DISCUSSION}

In the present study, threshold facilitation was found with primetarget identity (as in the word, word-and-outline, and outline transfer 
in Experiment 1). In some instances of prime-target alterations (i.e., outline-primed word thresholds; Experiment 1), enlarged words and brightness-reversed words as primes for words of normal size, and brightness-reversed (Experiment 2) and mirror-reversed primes (Experiment 3) preceding words of normal orientation also facilitated memory. It was only in Experiment 1, in which format shifts were introduced (i.e., for photograph-primed words, and word-primed and photographprimed outlines), that facilitation failed to occur.

We found more than one basis for facilitation, but encoding effects seemed to be at work in cases in which facilitation occurred despite prime-target alterations. One such case was the occurrence of facilitation of word thresholds by outline drawings, which appeared to result from the encoding entailed in naming the object depicted. The alterations introduced in Experiment 2-increased letter size and brightness reversal-did not change the overall envelope of the word, which made it an equivalent stimulus and produced the same encoding response as did the original word. The mirror-reversed words, introduced in Experiment 3, would seem to effect a sharp departure in the words' appearance and format. Nonetheless, threshold facilitation was obtained with such primes, and their increased response latencies suggest an effortfulness in encoding that may, along with problem solving and imagery, be the basis for the resulting facilitation.

The present results point toward the possibility that the many features of a priming stimulus may evoke a variety of mental operations that affect the manner in which the information is stored and becomes available as a source of potential influence in subsequent memory tests. This statement implies that words or pictures, when presented as primes, may be processed in ways that can be placed on a continuum from totally passive (as with the $\mathbf{S}$ items in paired-associate learning) to active-stimulus analysis, possibly combined with problem solving (which seems to have occurred with the mirror-reversed words). Delineation of the points along such a continuum would certainly reveal that these are, as Kolers and Roediger (1984) have put it, true "procedures of the mind."

\section{REFERENCES}

Craik, F. I. M., \& LockHART, R. S. (1972). Levels of processing: A framework for memory research. Journal of Verbal Learning \& Verbal Behavior, 11, 671-684.

JACOBY, L. L., \& BROOKS, L. R. (1984). Nonanalytic cognition: Memory, perception and concept learning. In G. Bower (Ed.), The psychology of learning and motivation (Vol. 18, pp. 1-47). New York: Academic Press.

JACOBY, L. L., \& HAYMAN, C. A. G. (1987). Specific visual transfer in word identification: Effects of word shape. Journal of Experimental Psychology: Learning, Memory, \& Cognition, 13, 456-463.

Kolers, P. A., \& Roediger, H. A. (1984). Procedures of the mind. Verbal Learning \& Verbal Behavior, 23, 425-449.

MoRToN, J. (1979). Facilitation in word recognition: Experiments causing change in the logogen model. In P. A. Kolers, M. E. Wrolstad, \& H. Bouma (Eds.), Processing of visible language (Vol. 1, pp. 259268). Hillsdale, NJ: Erlbaum.

Ritchey, G. H. (1982). Pictorial detail and recall in adults and children. Journal of Experimental Psychology: Human Learning \& Memory, 6, 66-76.

RoEdiger, H. L., \& Blaxton, T. A. (1987). Retrieval modes produce dissociations in memory for surface information. In D. Gorfein \& R. R. Hoffman (Eds.), Memory and cognitive processes: The Ebbinghaus Centennial Conference (pp. 349-379). Hillsdale, NJ: Erlbaum.

Scarborough, D. L., Gerard, L., \& Cortese, C. (1979). Accessing lexical memory: The transfer of word repetition effects across task and modality. Memory \& Cognition, 7, 3-12.

SNODGRASS, J. G., \& VANDERWART, M. (1980). A standardized set of 260 pictures: Norms for name agreement, image agreement, familiarity, and visual complexity. Journal of Experimental Psychology: Learning \& Memory, 6, 174-215.

Sperber, R. D., McCauley, C., Ragain, R. D., \& Weil, C. M. (1979). Semantic priming effects on picture and word processing. Memory \& Cognition, 7, 339-345.

Tulving, E., Schacter, D. L., \& Stark, H. A. (1982). Priming effects in word-fragment completion are independent of recognition memory. Journal of Experimental Psychology: Learning, Memory, \& Cognition, 8, 336-342.

Tyler, S. W., Hertel, P. H., McCallum, M. C., \& Ellis, H. C. (1979). Cognitive effort and memory. Journal of Experimental Psychology: Human Learning, \& Memory, 5, 607-617.

Weldon, M. S., \& Roediger, H. L. (1987). Altering retrieval demands reverses the picture superiority effect. Memory \& Cognition, 15, 269-280.

WinNick, W. A., \& DANIEL, S. A. (1970). Two kinds of response priming in tachistoscopic recognition. Journal of Experimental Psychology, 84, 74-81.

WinNick, W. A., \& NACHBaR, S. (1967). Tachistoscopic thresholds following paired-associate learning. Journal of Verbal Learning \& Verbal Behavior, 6, 95-100.

(Manuscript received February 3, 1989.) 